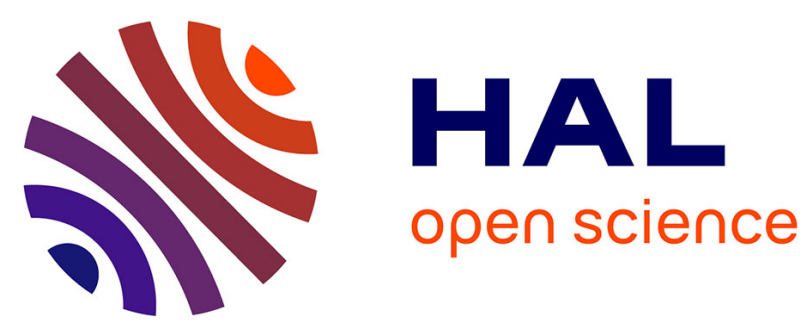

\title{
Intérêt de l'ostéodensitométrie chez les sujets chuteurs
}

Hubert Blain, Yves Rolland, Olivier Beauchet, Cédric Annweiler, Claude-Laurent Benhamou, Athanase Benetos, Gilles Berrut, Maurice Audran, Sauveur Bendavid, Valérie Bousson, et al.

\section{- To cite this version:}

Hubert Blain, Yves Rolland, Olivier Beauchet, Cédric Annweiler, Claude-Laurent Benhamou, et al.. Intérêt de l'ostéodensitométrie chez les sujets chuteurs. Revue du Rhumatisme, 2014, 81 (4), pp.297302. 10.1016/j.rhum.2014.01.004 . hal-01058268

HAL Id: hal-01058268 https://hal-univ-rennes1.archives-ouvertes.fr/hal-01058268

Submitted on 26 Aug 2014

HAL is a multi-disciplinary open access archive for the deposit and dissemination of scientific research documents, whether they are published or not. The documents may come from teaching and research institutions in France or abroad, or from public or private research centers.
L'archive ouverte pluridisciplinaire HAL, est destinée au dépôt et à la diffusion de documents scientifiques de niveau recherche, publiés ou non, émanant des établissements d'enseignement et de recherche français ou étrangers, des laboratoires publics ou privés. 


\section{Intérêt de l'ostéodensitométrie chez les sujets chuteurs}

Hubert Blain ${ }^{\mathrm{a},{ }^{*}}$, Yves Rolland ${ }^{\mathrm{b}}$, Olivier Beauchet ${ }^{\mathrm{c}}$, Cedric Annweiler ${ }^{\mathrm{c}}$, Claude-Laurent Benhamou $^{\mathrm{d}}$, Athanase Benetos ${ }^{\mathrm{e}}$, Gilles Berrut ${ }^{\mathrm{f}}$, Maurice Audran ${ }^{\mathrm{g}}$, Sauveur Bendavid ${ }^{\mathrm{h}}$, Valérie Bousson ${ }^{\mathrm{i}}$, Karine Briot ${ }^{\mathrm{j}}$, Michel Brazier ${ }^{\mathrm{k}}$, Véronique Breuil ${ }^{1}$, Laure Chapuis ${ }^{\mathrm{m}}$, Roland Chapurlat ${ }^{\mathrm{n}}$, Martine Cohen-Solal ${ }^{\mathrm{o}}$, Bernard Cortet $^{\mathrm{p}}$, Patricia Dargent ${ }^{\mathrm{q}}$, Patrice Fardellone $^{\mathrm{r}}$, Jean-Marc Feron ${ }^{\mathrm{s}}$, Jean-Bernard Gauvain ${ }^{\mathrm{t}}$, Pascal Guggenbuhl ${ }^{\mathrm{u}}$, Olivier Hanon ${ }^{\mathrm{v}}$, Michel Laroche ${ }^{\mathrm{w}}$, Sami Kolta ${ }^{\mathrm{j}}$, Eric Lespessailles ${ }^{\mathrm{r}}$, Brigitte Letombe ${ }^{\mathrm{x}}$, Eric Mallet ${ }^{\mathrm{y}}$, Christian Marcelli $^{\mathrm{z}}$, Philippe Orcel ${ }^{\mathrm{aa}}$, François Puisieux ${ }^{\mathrm{ab}}$, Patrick Seret ${ }^{\mathrm{ac}}$, Jean-Claude Souberbielle ${ }^{\mathrm{ad}}$, Bruno Sutter $^{\mathrm{ae}}$, Florence Trémollières ${ }^{\mathrm{af}}$, Georges Weryha ${ }^{\mathrm{ag}}$, Christian Roux ${ }^{\text {ah }}$, Thierry Thomas $^{\mathrm{ag}}$. Pour le Groupe de Recherche et d'Information sur les Ostéoporoses et la Société Française de Gérontologie et Gériatrie.

\footnotetext{
vieillissement actif en Languedoc Roussillon France,

b Gérontopôle de Toulouse, Hôpital La Grave-Casselardit, CHU Toulouse, France

c UPRES EA 4638,Service de gériatrie, CHU Angers, France

d EA4708 I3MTO, University of Orleans, France.

e Service de Gériatrie, CHU Nancy, Inserm U1116, Université de Lorraine, France

f Service de gériatrie, CHU Nantes, France

g Service de rhumatologie et GEROM, CHU d'Angers, France

h Médecine Générale, Paris, France

i Service de Radiologie Ostéo-Articulaire, Hôpital Lariboisière, Paris, France

j Service de rhumatologie, hôpital Cochin, université Paris-Descartes, Paris, France

k Service de rhumatologie, Hôpital Nord, Amiens, France.

If Service de rhumatologie, CHU de Nice-1, université Nice Sophia-Antipolis, Nice, France

m Service de rhumatologie, 35500 Vitré, France

n Serice de rhumatologie, CHU Lyon, France

o INSERM U606, Université Paris-Diderot Paris 7, hôpital Lariboisière, Paris, France

p EA 4490, service de rhumatologie, CHU de Lille, Lille, France

q Université Paris-Sud, UMRS 1018, Villejuif, France

r Inserm ERI 12, service de rhumatologie, CHU d'Amiens, Amiens, France

s Service de chirurgie orthopédique, hôpital Saint-Antoine, Paris, France

t Centre de médecine gériatrique, CHR d'Orléans, Orléans, France

u, Inserm UMR U 991, Service de rhumatologie, CHU de Rennes, Rennes, France

v Service de gérontologie, hôpital Broca, Paris, France;

w Centre de Rhumatologie, CHU Purpan, Toulouse, France

x Service de gynécologie médicale et médecine du couple, hôpital Jeanne-de-Flandre, CHRU de Lille, Lille, France

y Centre de référence des maladies rares du calcium et du phosphore, CIC Inserm 204, CHU de Rouen, Rouen, France

z Service de rhumatologie, hôpital Côte-de-Nacre, Caen, France

aa Service de rhumatologie, hôpital Lariboisière, Paris, France

ab. Servive de Gériatrie, CHU Lille, France.

ac Service de rhumatologie, Angers, France

ad Laboratoire d'Explorations Fonctionnelles, et INSERM U845, Hôpital Necker-Enfants Malades, Paris, France

ae Service de chirurgie orthopédique des adultes de l'Institut Calot de Berck, France

af Centre de ménopause, hôpital Paule-de-Viguier, Toulouse, France

ag Service d'endocrinologie, CHU de Nancy, Vandoeuvre-lès-Nancy, France

ahINSERM U1059, Service de Rhumatologie, CHU de Saint-Etienne, 42055 Saint-Etienne, Cedex 2, France
}

a Pôle de Gériatrie, Centre Antonin Balmes, CHU Montpellier,39 avenue Charles Flahault, 34395 Montpellier Cedex 5r, France; Laboratoire Movement to Health, Euromov, 700 avenue du Pic Saint-Loup, 34090 Montpellier, Université Montpellier 1, Site de Référence MACVIA-LR, Contre les Maladies Chroniques pour un

*Auteur correspondant. Fax:+33 467336790

Adresse e-mail: h-blain@chu-montpellier.fr

Mots clés : Ostéoporose; ostéodensitométrie ; chute, prise en charge ; recommandations ; fragilité 


\section{R E S U M E}

La présente revue de la littérature a pour objectif d'argumenter les nouvelles recommandations françaises publiées en 2012 retenant le risque de chute, évalué par l'antécédent de chute dans la dernière année, comme indication de la mesure de la densité minérale osseuse (DMO) par l'absorptiométrie biphotonique par rayons X (DXA). Cette recommandation repose sur le fait que 1. L'ostéoporose et le risque de chute représentent les deux premiers facteurs de risque de fracture non vertébrale après la ménopause. 2. La mesure de la DMO par DXA apporte une information significative sur le risque de fracture, indépendamment du risque de chute. Ainsi, un sujet chuteur aura d'autant plus de risque de se fracturer qu'il aura une DMO basse. 3. Les traitements anti-ostéoporotiques n'ont montré une efficacité anti-fracturaire qu'en cas d'ostéoporose ostéodensitométrique. 4. La prévalence de l'ostéoporose est élevée chez les sujets chuteurs d'autant que ceux-ci sont fragiles [chutes répétées, sarcopénie (faible masse et force musculaire), réduction de mobilité, perte de poids, en particulier)], ces facteurs étant des facteurs de risque communs d'ostéoporose et de chute.

Cependant, l'indication de la DXA doit être nuancée chez les sujets chuteurs dont l'espérance de vie est limitée, les traitements n'ayant montré une efficacité anti-fracturaire qu'après 12 mois d'administration et en cas de faible accessibilité à la DXA (éloignement, patients dépendants, ayant une altération cognitive sévère par exemple). Des travaux sont souhaitables pour mieux définir comment intégrer le risque de chute et la fragilité dans l'évaluation du risque de fracture par la DMO et le score FRAX ${ }^{\circledR}$. 
L'ostéoporose est une maladie diffuse du squelette touchant particulièrement les femmes ménopausées, caractérisée par une réduction de la résistance osseuse conduisant à un risque accru de fracture [1]. L'ostéodensitométrie (DXA), qui mesure la densité minérale osseuse (DMO), est actuellement le meilleur outil pour identifier les patients à risque de fracture en raison d'une masse osseuse basse. Malgré son remboursement en France depuis 2006 en présence d'un ou plusieurs facteurs de risque clinique (publication du Journal Officiel du 30 juin 2006 - Annexe 1) [2], le nombre de DXA réalisées tend à diminuer en France [3], en l'absence de réduction de l'incidence des fractures non vertébrales [4], suggérant un défaut de prise en charge des malades à haut risque de fracture qui justifient d'un traitement antiostéoporotique.

De manière à adapter les recommandations de prise en charge de l'ostéoporose postménopausique aux nouvelles connaissances concernant les facteurs de risque et de prévention des fractures, la Société Française de Rhumatologie, le Groupe de Recherche et d'Information sur les Ostéoporoses, le Collège National des Gynécologues et Obstétriciens Français, le Groupe d'Etude de la Ménopause et du Vieillissement hormonal, la Société Française de Chirurgie Orthopédique, la Société Française d'Endocrinologie et la Société Française de Gériatrie et de Gérontologie ont redéfini en 2012 les indications de la DXA et des traitements anti-ostéoporotiques [6]. Dans ces recommandations 2012, la survenue d'une fracture sévère, responsable d'un risque élevé de mortalité, notamment les fractures de vertèbres, du fémur, du bassin, et de l'humérus [7], constitue une indication à prescrire un traitement antiostéoporotique en l'absence de contre-indication ou d'autre cause de fragilité osseuse. La DXA n'est plus, dans ce cas, un élément de la décision thérapeutique, même si elle reste un élément important dans le monitoring des malades.

La DXA reste en revanche déterminante dans la décision thérapeutique en présence d'un facteur de risque clinique autre qu'une fracture sévère (Tableau 1). Les recommandations de 
2012 proposent d'inclure dans cette démarche les femmes ménopausées à risque significatif de chute, en particulier en cas d'antécédent de chute dans la dernière année, premier facteur de risque de chute incidente $[8,9]$.

Le présent article a pour objectif d'argumenter la recommandation de 2012 d'effectuer une DXA chez les sujets chuteurs, l'ostéodensitométrie n'étant cependant pour l'heure pas remboursée en France dans cette indication.

\section{Liens entre ostéoporose et chutes chez les femmes ménopausées}

Les femmes ostéoporotiques âgées de 60 ans ou plus présentent deux fois plus d'antécédents de chute dans la dernière année que les femmes non ostéoporotiques [10].

Ce lien entre ostéoporose et chute s'explique en partie par un certain nombre de déterminants communs, incluant un âge élevé, certains paramètres génétiques et anthropométriques (perte de poids, faible masse musculaire en particulier), des niveaux faibles de force musculaire, d'activité physique, de mobilité et certaines déficiences hormonales (concernant en particulier l'axe GH/IGF1, la vitamine D et les hormones sexuelles) [11-20].

En outre, l'ostéoporose, lorsqu'elle est compliquée de fractures vertébrales responsables d'une cyphose dorsale, peut entraîner des modifications d'équilibre, de force musculaire (préhension, extension du tronc, et des membres inférieurs), des douleurs rachidiennes, une peur de tomber, qui sont autant de facteurs de risque de chute [10, 21-23].

A l'inverse, la prévalence de l'ostéoporose n'est pas accrue chez les femmes ménopausées chuteuses [24]. Ceci est probablement expliqué par la multiplicité des causes de chute chez les sujets âgés, impliquant des facteurs de risque n'ayant pas d'influence directe sur la densité osseuse, tels qu'une vision diminuée par exemple [9].

$\mathrm{Au}$ total, si ces données montrent qu'ostéoporose et risque de chute partagent un certain nombre de déterminants communs, justifiant de les rechercher de manière conjointe, il ne faut 
pas considérer que tous les sujets chuteurs sont ostéoporotiques et relèvent systématiquement d'un traitement anti-ostéoporotique. La mesure d'une DXA avant de décider d'un traitement anti-ostéoporotique chez les sujets chuteurs est d'autant plus nécessaire qu'aucune donnée n'est disponible suggérant qu'un traitement peut réduire le risque de fracture dans une population uniquement sélectionnée sur un risque de chute $[25,26]$. La présence d'une ostéoporose ostéodensitométrique ou fracturaire est donc un pré-requis pour qu'un traitement anti-ostéoporotique réduise significativement le risque de fracture [26-29].

\section{La mesure de la DMO par DXA apporte-t-elle une information sur le risque de} fracture chez les sujets chuteurs?

Chez les femmes ménopausées non sélectionnées, le facteur de risque de fracture le plus significatif est la réduction de la DMO appréciée par DXA [30]. Le risque de fracture dépend d'autres paramètres osseux, dont la géométrie, la micro-architecture et le remodelage, difficiles à intégrer dans le cadre d'un dépistage à grand échelle des sujets à risque de fracture [12]. Outre ces paramètres évaluant la résistance osseuse, le risque de fracture d'un segment osseux dépend des contraintes mécaniques qui vont s'exercer sur lui. Le recouvrement important des valeurs de DMO des patients avec ou sans fracture [31] et le fait que près de $50 \%$ des patients avec fracture de hanche ne présentent pas d'ostéoporose ostéodensitométrique [32, 33] s'expliquent en partie par le fait que seuls les patients rencontrant une situation de contrainte osseuse d'intensité dépassant la résistance mécanique de leur squelette vont présenter une fracture.

La chute de sa hauteur représente de loin la première cause de traumatisme de la vie courante chez les femmes ménopausées en France, touchant environ 30\% des femmes de plus de 65 ans et plus de $40 \%$ des femmes de plus de 75 ans [34]. Cette donnée épidémiologique 
explique pourquoi la chute de sa hauteur représente l'une des principales causes de fracture non vertébrale [35].

Plusieurs études ont montré que la mesure de la DMO apporte une information significative sur le risque de fracture chez des femmes ménopausées vivant au domicile, et ceci indépendamment de la prise en compte des antécédents et des facteurs de risque de chute, tels que la dysfonction neuromusculaire [36], les troubles de la mobilité [36], un équilibre précaire [11], des troubles cognitifs [37], une vision altérée [11], et la consommation de médicaments sédatifs [12], en particulier.

En établissement hébergeant des sujets âgés dépendants (EHPAD), les sujets sont le plus souvent à risque de chute, l'incidence annuelle des chutes étant estimée à 1,5 par lit en moyenne [38, 39]. Les chutes sont des chutes répétées pour plus de la moitié des résidents [40]. Comme pour les sujets vivant à domicile, le risque de fracture de hanche est accru chez les sujets à DMO basse, indépendamment de leur mobilité et du type de contrainte exercée par la chute [41].

L'ensemble de ces données suggèrent l'intérêt de mesurer la DMO pour évaluer le risque de fracture des sujets à risque de chute ou chuteurs, vivant au domicile ou en EHPAD. La mesure de la DMO par DXA peut en revanche se discuter pour les patients chez lesquels l'accessibilité de l'examen est difficile (patients les plus dépendants, ayant des fonctions cognitives altérées, ou dont le lieu d'habitation est éloigné de l'ostéodensitomètre).

\section{Existe-t-il un intérêt à proposer une DXA chez les femmes ménopausées chuteuses}

\section{fragiles ?}

Comme indiqué précédemment, l'ostéoporose sera plus fréquemment observée chez un sujet âgé chuteur présentant des facteurs de risque communs à la chute et à l'ostéoporose, tels qu'un âge élevé, une perte de poids, un faible poids, des niveaux faibles de masse musculaire et de 
force musculaire (sarcopénie), d'activité physique et de mobilité, les contraintes mécaniques étant essentielles pour la maintenance de la masse osseuse [12-18]. Ces paramètres sont des indicateurs de fragilité du sujet âgé, ce concept récent de fragilité du sujet âgé étant défini comme un état de vulnérabilité et de mauvaise capacité d'adaptation en cas d'agression, à l'origine d'un cercle vicieux d'évènements négatifs de santé incluant, outre la chute et ses conséquences fracturaires, la perte d'autonomie, l'institutionnalisation et le décès [42-44].

Le lien étroit entre un niveau élevé de fragilité et une DMO basse chez les sujets âgés est observé dans plusieurs études [45, 46]. Les sujets âgés fragiles, présentant des chutes répétées, ont une DMO plus basse que les non chuteurs [47], suggérant l'intérêt tout particulier de rechercher une ostéoporose quand la chute du sujet s'intègre dans un contexte de chutes répétées et de fragilité [16]. En outre, la prise en compte des facteurs de fragilité, indépendamment de la DMO, apporte une information significative sur le risque de fracture [48]. Enfin, une étude a montré que le nombre de sujets nécessaires à traiter pour prévenir un évènement fracturaire pourrait être plus faible chez les femmes ménopausées ostéoporotiques fragiles par rapport aux femmes ménopausées ostéoporotiques non fragiles [49].

L'intérêt du concept de fragilité repose sur son caractère potentiellement réversible en cas de prise en charge de ses différentes causes et conséquences potentielles, dont la chute et la fracture ostéoporotique [43, 44]. Ainsi, la recherche et la prise en charge de l'ostéoporose ne sera qu'un des éléments de prise en charge des patients chuteurs fragiles ostéoporotiques, une prise en charge multi-dimensionnelle étant seule capable de prévenir le "cercle vicieux" induit par la sarcopénie, les chutes, la limitation d'activité, de dépense énergétique et des apports nutritionnels qui vont, sinon, entraîner à leur tour une perte de poids et aggraver la sarcopénie [44]. 


\section{La découverte d'une ostéoporose chez une femme ménopausée chuteuse modifie-t-elle la prise en charge non médicamenteuse ?}

Un certain nombre de mesures permettent de réduire l'incidence de nouvelles chutes chez les sujets âgés chuteurs. Ces mesures comportent en particulier l'aménagement du domicile, au mieux proposé par un ergothérapeute, et ce, d'autant que les sujets sont à haut risque de chute et ont des troubles visuels. Ces mesures comportent aussi le dépistage de la cataracte et son traitement chirurgical si nécessaire, le remplacement de verres multi-focaux par des verres progressifs en cas de chutes extérieures au domicile, le sevrage progressif en médicaments favorisant la chute, dont les psychotropes, la correction d'une carence ou une insuffisance en vitamine D (recommandations Haute Autorité de Santé)(http://www.hassante.fr/portail/jcms/c_1356838/fr/utilite-clinique-du-dosage) et la pose d'un pace-maker en cas d'hypersensibilité du sinus carotidien [19, 50]. Les programmes d'activité physique adaptée, comportant un travail de l'équilibre et au moins un autre type d'exercices, incluant un renforcement musculaire, un travail de la coordination, et/ou de l'endurance ont montré une efficacité à réduire l'incidence des chutes et leurs complications fracturaires [50, 51].

En outre, si les programmes visant à améliorer l'équilibre, la coordination et l'endurance réduisent l'incidence des chutes, ces programmes n'ont pas d'effet significatif sur la DMO, contrairement aux programmes incluant des exercices en charge, induisant des contraintes mécaniques osseuses d'intensité et de durée suffisantes, tels que des sauts ou, plus réalisables chez les sujets âgés, un renforcement musculaire en regard des segments osseux à solliciter $[\underline{52,53]}$. Quand ces mesures conjointes sont effectuées chez des femmes âgées de 65 ans ou plus ayant une DMO basse, ces mesures permettent de réduire les chutes et d'influencer favorablement la DMO, à condition que ces programmes aient une durée supérieure à 6 mois [45, 54-56]. En cas d'ostéoporose avec cyphose, un renforcement musculaire des extenseurs 
du tronc induit une amélioration de la mobilité du tronc et des douleurs rachidiennes, facteurs importants pour prévenir les chutes [22, 23].

\section{Conclusion}

La mesure par la DXA de la DMO a récemment été recommandée en cas d'antécédent de chute par un groupe d'experts en France, rejoignant des recommandations du UK National Service Framework [57]. Cette recommandation repose sur le fait que la mesure de la DMO chez un sujet chuteur apporte une information significative sur le risque de fracture, indépendamment des autres facteurs de risque de chute et que les traitements antiostéoporotiques n'ont montré une efficacité anti-fracturaire qu'en cas d'ostéoporose ostéodensitométrique ou fracturaire. L'ostéoporose sera d'autant plus souvent présente lors de la mesure de la DMO par DXA que la chute s'intègre dans le cadre d'une fragilité plus générale, la sarcopénie, la réduction de mobilité et la perte de poids étant des déterminants communs à la chute et à l'ostéoporose. Dans ce cas, la prise en charge comportera non seulement un traitement anti-ostéoporotique mais aussi une prise en charge multidimensionnelle visant à prévenir une nouvelle chute et ciblant les déterminants et les autres conséquences potentielles de la fragilité. Il faudra cependant discuter l'indication de la DXA chez les sujets chuteurs dont l'espérance de vie est limitée, les traitements n'ayant montré une efficacité anti-fracturaire qu'après 12 mois $[49,58,59]$. Enfin, des travaux sont souhaitables pour mieux définir comment intégrer le risque de chute et la fragilité dans l'évaluation du risque de fracture évalué par la DMO et le score de risque de fracture $\operatorname{FRAX}{ }^{\circledR}(60,61)$. Même si le présent argumentaire plaide en faveur de la réalisation d'une ostéodensitométrie à $\underline{\text { la recherche d'une ostéoporose chez les sujets âgés à haut risque de chute, cet examen n'est }}$ pour l'heure pas remboursé dans cette indication. 
Tableau 1 : Indications du remboursement de l'ostéodensitométrie (DXA) retenues par Agence Française de Sécurité Sanitaire des Produits de Santé en 2006 http://www.grio.org/documents/rcd-3-1263309626.pdf)

\section{Indications de remboursement de la DXA retenues en 2006 dans la population} générale

- en cas de signes d'ostéoporose :

* découverte ou confirmation radiologique d'une fracture vertébrale (déformation du corps vertébral) sans contexte traumatique ni tumoral évident

* antécédent personnel de fracture périphérique survenue sans traumatisme majeur (sont exclues de ce cadre les fractures du crâne, des orteils, des doigts et du rachis cervical) - en cas de pathologie ou traitement potentiellement inducteur d'ostéoporose :

* lors d'une corticothérapie systémique (de préférence au début) prescrite pour une durée d'au moins 3 mois consécutifs, à une dose $>7,5 \mathrm{mg}$ /jour d'équivalent prednisone * antécédent documenté de pathologie ou de traitement inducteur d'ostéoporose : hyperthyroïdie évolutive non traitée, hypercorticisme, hyperparathyroïdie primitive hypogonadisme prolongé (incluant l'androgénoprivation chirurgicale [orchidectomie] ou médicamenteuse [traitement prolongé par un analogue de la Gn-RH]) et ostéogenèse imparfaite

\section{Indications complémentaires de remboursement de la DXA retenues en 2006 chez la} femme ménopausée

- antécédents de fracture du col fémoral sans traumatisme majeur chez un parent au 1er degré - indice de masse corporelle $<19 \mathrm{~kg} / \mathrm{m}^{2}$

- ménopause avant 40 ans quelle qu'en soit la cause

- antécédent de corticothérapie prolongée (> 3 mois) à la dose de corticoïde équivalent prednisone $\geq 7,5 \mathrm{mg}$ par jour 


\section{Références}

1. NIH Consensus Development Panel on Osteoporosis. JAMA 2001;285:785-95.

2. http://www.Afssaps-sante.fr/portail/upload/docs/application/pdf/osteoporose_synthese.pdf

3. Canoui-Poitrine F, Jaglal S, Chapurlat R, et al. Afssaps reimbursement of bone mineral density testing and anti-osteoporotic treatments improved management of osteoporosis in France? Bone 2010;47:790-4.

4.Curran D, Maravic M, Kiefer P, et al. Epidemiology of osteoporosis- related fractures in France: a literature review. Joint Bone Spine 2010;77:546-51.

5. Maravic M, Taupin P, Landais P, Roux C. Changement dans l'incidence des fractures de la hanche au cours des 6 dernières années en France. Osteoporos Int 2011;22:797-801.

6. Briot K, Cortet B, Thomas T, et al. 2012 update of French guidelines for the pharmacological treatment of postmenopausal osteoporosis. Joint Bone Spine 2012;79:30413.

7. Bliuc D, Nguyen ND, Milch VE, et al. Mortality risk associated with low-trauma osteoporotic fracture and subsequent fracture in men and women. JAMA 2009;301:513-21.

8. Ganz DA, Bao Y, Shekelle PG, Rubenstein LZ. Will my patient fall? JAMA 2007;297:7786.

9. Clinical Practice Guideline for the Assessment and Prevention of Falls in Older People. National Collaborating Centre for Nursing and Supportive Care (UK). London: Royal College of Nursing (UK); 2004 Nov. http:/www.nice.org.uk.

10. da Silva RB, Costa-Paiva L, Morais SS, et al. Predictors of falls in women with and without osteoporosis. J Orthop Sports Phys Ther 2010;40:582-8.

11. Dargent-Molina P, Favier F, Grandjean H et al. Fall-related factors and risk of hip fracture: the EPIDOS prospective study. Lancet 1996;348:145-9.

12. Allolio B. Risk factors for hip fracture not related to bone mass and their therapeutic implications. Osteoporos Int 1999;9:S9-S16.

13. Pijnappels M, van der Burg PJ, Reeves ND, van Dieën JH. Identification of elderly fallers by muscle strength measures. Eur J Appl Physiol 2008;102:585-92.

14. Buatois S, Miljkovic D, Manckoundia P, et al. Five times sit to stand test is a predictor of recurrent falls in healthy community-living subjects aged 65 and older. J Am Geriatr Soc 2008;56:1575-7.

15. Perrini S. The GH/IGF1 axis and signaling pathways in the muscle and bone: mechanisms underlying age-related skeletal muscle wasting and osteoporosis J Endocrinol 2010;205:20110.

16. Frisoli A Jr, Chaves PH, Ingham SJ, Fried LP. Severe osteopenia and osteoporosis, sarcopenia, and frailty status in community-dwelling older women: results from the Women's Health and Aging Study (WHAS) II. Bone 2011;48:952-7.

17. Sirola J, Kröger H. Similarities in acquired factors related to postmenopausal osteoporosis and sarcopenia. J Osteoporos 2011;2011:536735.

18. Goldspink G. Age-Related Loss of Muscle Mass and Strength. J Aging Res 2012;2012:158279.

19. Rizzoli R, Boonen S. Vitamin D supplementation in elderly or postmenopausal women: a 2013 update of the 2008 recommendations from the European Society for Clinical and Economic Aspects of Osteoporosis and Osteoarthritis (ESCEO). Curr Med Research 2013;29:305-13.

20. Lynn SG, Sinaki M, Westerlind KC. Balance characteristics of persons with osteoporosis. Arch Phys Med Rehabil 1997;78:273-7. 
21. Arnold CM, Busch AJ, Schachter CL, et al. The relationship of intrinsic fall risk factors to a recent history of falling in older women with osteoporosis. J Orthop Sports Phys Ther 2005;35:452-60.

22. Sinaki M, Brey RH, Hughes CA, et al. Significant reduction in risk of falls and back pain in osteoporotic-kyphotic women through a Spinal Proprioceptive Extension Exercise Dynamic (SPEED) program. Mayo Clin Proc 2005;80:849-55.

23. Chien MY, Yang RS, Tsauo JY. Home-based trunk-strengthening exercise for osteoporotic and osteopenic postmenopausal women without fracture--a pilot study. Clin Rehabil 2005;19:28-36.

24. Newton JL, Jones DE, Wilton K, et al. Calcaneal bone mineral density in older patients who have fallen. QJM 2006;99:231-6.

25. Siris ES, Simon JA, Barton IP, et al. Effects of risedronate on fracture risk in postmenopausal women with osteopenia. Osteoporos Int 2008;19:681-6.

26. Cummings SR, Black DM, Thompson DE, et al. Effect of alendronate on risk of fracture in women with low bone density but without vertebral fractures: results from the Fracture Intervention Trial. JAMA 1998;280:2077-82.

27. Kanis JA, Johnell O, Black DM, et al. Effect of raloxifene on the risk of new vertebral fracture in postmenopausal women with osteopenia or osteoporosis: a reanalysis of the Multiple Outcomes of Raloxifene Evaluation trial. Bone 2003;33:293-300.

28. Ryder KM, Cummings SR, Palermo L, et al. Fracture Intervention Trial Research Group. Does a history of non-vertebral fracture identify women without osteoporosis for treatment? J Gen Intern Med 2008;23:1177-81.

29. Donaldson MG, Palermo L, Ensrud KE, et al. Effect of alendronate for reducing fracture by FRAX score and femoral neck bone mineral density: the Fracture Intervention Trial. J Bone Miner Res 2012;27:1804-10.

30. Marshall D, Johnell O, Wedel H. Meta-analysis of how well measures of bone mineral density predict occurrence of osteoporotic fracture. BMJ 1996;312:1254-9.

31. Ross PD, Davis JW, Vogel JM, Wasnich RD. A critical review of bone mass and the risk of fractures in osteoporosis. Calcif Tissue Int 1990;46:149-61.

32. Black DM, Steinbuch M, Palermo L, et al. An assessment tool for predicting fracture risk in postmenopausal women. Osteoporos Int 2001;12:519-28.

33. Lyles KW, Colon-Emeric CS, Magaziner SJ, et al. Zoledronic acid in reducing clinical fracture and mortality after hip fracture. N Eng J Med 2007;357:nihpa40967.

34. Numéro thématique - Épidémiologie et prévention des chutes chez les personnes âgées (Special issue - Epidemiology and prevention of falls among the elderly). Bulletin épidémiologique hebdomadaire. 2007 (2 octobre); 37-8.

35. Edwards MH, Jameson K, Denison $\mathrm{H}$, et al. Clinical risk factors, bone density and fall history in the prediction of incident fracture among men and women. Bone 2013;52:541-7.

36. Wei TS, Hu CH, Wang SH, Hwang KL. Fall characteristics, functional mobility and bone mineral density as risk factors of hip fracture in the community-dwelling ambulatory elderly. Osteoporos Int 2001;12:1050-5.

37. Härlein J, Dassen T, Halfens RJ, Heinze C. Fall risk factors in older people with dementia or cognitive impairment: a systematic review. J Adv Nurs 2009;65:922-33.

38. Cameron ID, Gillespie LD, Robertson MC, et al. Interventions for preventing falls in older people in care facilities and hospitals. Cochrane Database Syst Rev 2012;12:CD005465.

39. Société Française de Gérontologie et Gériatrie - Haute Autorité de Santé / Service des recommandations professionnelles, Recommandations pour la pratique clinique. Évaluation et prise en charge des personnes âgées faisant des chutes répétées. Avril 2009 
40. SFDRMG - HAS / Service des recommandations professionnelles, Recommandations pour la pratique clinique Prévention des chutes accidentelles chez la personne âgée. Argumentaire novembre 2005.

41. Greenspan SL, Myers ER, Kiel DP, et al. Fall direction, bone mineral density, and function: risk factors for hip fracture in frail nursing home elderly. Am J Med 1998;104:53945.

42. Fried LP, Tangen CM, Walston J, et al; Cardiovascular Health Study Collaborative Research Group. Frailty in older adults: evidence for a phenotype. J Gerontol A Biol Sci Med Sci 2001;56:M146-56.

43. Cesari M, Leeuwenburgh C, Lauretani F, et al. Frailty syndrome and skeletal muscle: results from the Invecchiare in Chianti study. Am J Clin Nutr 2006;83:1142-48.

44. Ko FC. The clinical care of frail, older adults. Clin Geriatr Med 2011;27:89-100.

45. Crepaldi G, Maggi S. "Sarcopenia and osteoporosis: a hazardous duet". J Endocrinol Investigation 2005;28: 66-68.

46. Rolland Y, Abellan Van Kan G, Benetos A, et al., "Frailty, osteoporosis and hip fracture: causes, consequences and therapeutic perspectives". J Nutr Health Aging 2008;12:335-346.

47. Newton JL, Kenny RA, Frearson R, francis RM. A prospective evaluation of bone mineral density measurement in females who have fallen. Age Ageing 2003;32:497-502.

48. Cheung EY, Bow CH, Cheung CL, et al. Discriminative value of FRAX for fracture prediction in a cohort of Chinese postmenopausal women. Osteoporos Int 2012;23:871-8.

49. Rolland Y, Van Kan GA, Gillette-Guyonnet S, et al. Strontium ranelate and risk of vertebral fractures in frail osteoporotic women. Bone 2011;48:332-8.

50. Gillespie LD, Robertson MC, Gillespie WJ, et al. Interventions for preventing falls in older people living in the community (Review). The Cochrane Library 2012;9:CD007146.

51. El-Khoury F, Cassou B, Charles MA, Dargent-Molina P. The effect of fall prevention

exercise programmes on fall induced injuries in community dwelling older adults: systematic review and meta-analysis of randomised controlled trials. BMJ 2013;347:f6234.

52. Englund U, Littbrand H, Sondell A, et al. The beneficial effects of exercise on BMD are lost after cessation: a 5-year follow-up in older post-menopausal women. Scand J Med Sci Sports 2009;19:381-8.

53. Karinkanta S, Heinonen A, Sievänen H, et al. Maintenance of exercise-induced benefits in physical functioning and bone among elderly women.Osteoporos Int 2009;20:665-74.

54. Chodzko-Zajko WJ, Proctor DN, Fiatarone Singh MA, et al. American College of Sports Medicine position stand. Exercise and physical activity for older adults. Med Sci Sports Exerc 2009;41:1510-30.

55. Oliver D. Development of services for older patients with falls and fractures in England: successes, failures, lessons and controversies. Arch Gerontol Geriatr 2009;49:S7-S12.

56. Marques EA, Mota J, Carvalho J. Exercise effects on bone mineral density in older adults: a meta-analysis of randomized controlled trials. Age (Dordr). 2012;34:1493-515.

57. National Service Framework[http://www.dh.gov.uk/prod_consum_dh/groups/dh_digitalassets/@dh/@en/docu ments/digitalasset/dh_4071283.pdf].

58. Wells GA, Cranney A, Peterson J, et al. Alendronate for the primary and secondary prevention of osteoporotic fractures in postmenopausal women. Cochrane Database Syst Rev. 2008;1:CD001155.

59. Gallagher JC, Genant HK, Crans GG, et al. Teriparatide reduces the fracture risk associated with increasing number and severity of osteoporotic fractures. J Clin Endocrinol Metab 2005;90:1583-7. 
60. Kanis JA, McCloskey EV, Johansson H, et al; National Osteoporosis Guideline Group. Case finding for the management of osteoporosis with FRAX ${ }^{\circledR}-$ assessment and intervention thresholds for the UK. Osteoporos Int 2008;19:1395-1408. (www.shef.ac.uk/FRAX)

61. Masud T, Binkley N, Boonen S, Hannan MT; FRAX(®) Position Development Conference Members. Official Positions for FRAX® clinical regarding falls and frailty: can falls and frailty be used in FRAX®? From Joint Official Positions Development Conference of the International Society for Clinical Densitometry and International Osteoporosis Foundation on FRAX®.J Clin Densitom 2011;14:194-204. 